\title{
EL ENTORNO DE LA ORGANIZACIÓN: UN ESTUDIO DE SUS TIPOLOGÍAS Y SU VINCU- LACIÓN CON LA PERCEPCIÓN DIRECTIVA Y EL DISEÑO ORGANIZATIVO
}

The Organizational Environment: A Study of the Environmental Types and their links with the Managers' Perception and Organizational Design

\section{Gerardo J . Zapata Rotundo* Alberto Mirabal** María Teresa Canet Giner***}

\begin{abstract}
Resumen: Esta investigación de carácter teórico tiene como objetivo estudiar diferentes tipologías de entornos organizacionales y su vinculación con la percepción directiva y el diseño organizativo bajo dos perspectivas: control externo y voluntarismo. En la primera, se ubican las teorías contingente y dependencia de recursos, y en la segunda la teoría de la strategic choice. De su análisis, surgen un conjunto de proposiciones que podrán ser contrastadas en estudios posteriores. Como reflexión relevante, se aprecia que los niveles
\end{abstract}

\footnotetext{
Doctor en Dirección de Empresas por la Universidad de Valencia, España. Profesor Titular del Decanato de Administración y Contaduría de la Universidad Centroccidental "Lisandro Alvarado", Venezuela. Correo electrónico: zapager@yahoo.com

** Doctor en Dirección de Empresas por la Universidad de Valencia, España. Profesor Titular del Decanato de Administración y Contaduría de la Universidad Centroccidental "Lisandro Alvarado", Venezuela. Correo electrónico: albertomirabal@ucla.edu.ve

*** Doctora en Ciencias Económicas y Empresariales. Profesora Titular de la Universidad de Valencia-España, Departamento de Dirección de Empresas de la Facultad de Economía de la Universidad de Valencia, España. Correo electrónico: teresa.canet@uv.es
} 
de percepción de las condiciones y características del entorno por quienes dirigen a la organización, contribuye a la formación de su visión sobre la manera como debe ser diseñada y puesta en marcha las estrategias más adecuadas a los requerimientos de ese entorno.

Palabras clave: diseño organizativo, entorno, percepción directiva, tipología de entornos, sesgos cognitivos.

Abstract: The aim of this theoretical research is to study different types of organizational environment and its links with the managers' perception and organizational design from two perspectives: external control and voluntarism. In the first of these perspectives are located the contingent and resource dependency theories, and in the second the theory of strategic choice. Derived from the analysis, arise a set of propositions that can be tested in subsequent studies. As a relevant reflection, the article proves that the levels of perception that managers have about the conditions and characteristics of the organizational environment, should contribute to the formation of their vision about the way strategies should be designed and appropriately implemented to adapt to the requirements of the environment.

Keywords: organizational design, environments, directive perception, typology of environments, cognitive biases. 
El entorno de la organización: un estudio de sus tipologías y su vinculación con la percepción directiva y el diseño organizativo

\section{Introducción}

Un entorno cada vez más competitivo, trae consigo amenazas y oportunidades que implican incorporar procesos estratégicos a la organización que le permitan alcanzar un adecuado ajuste entre las variables internas de diseño organizativo ${ }^{1}$, con las variables externas del entorno donde se desenvuelve. Pero todo ello, depende en gran medida de la percepción e interpretación de los directivos y gerentes sobre esos condicionantes. Es decir, la traducción de los eventos del entorno conduce al desarrollo de modelos y esquemas conceptuales que permiten comprenderlo y en consecuencia, poner en práctica soluciones a determinados problemas a los que la organización se enfrenta, ideas éstas que pueden ser desarrolladas fundamentalmente bajo dos perspectivas teóricas: el control externo y el voluntarismo.

La perspectiva de control externo establece que las actividades, los recursos, el diseño organizativo y los productos que elabora la organización se explican a partir de la comprensión y satisfacción de los factores y condicionantes del entorno donde se desenvuelve (Pfeffer y Salancik, 1978; Rao, 2007; Nienhüser, 2008; Schwalb, Leavitt, Barbuto, Millard y Peetz, 2011; Zapata y Mirabal, 2011; Kloviene, 2013), siendo principalmente a través de la teoría contingente y la teoría de dependencia de recursos que se puede abordar esas consideraciones.

La Teoría Contingente plantea la proposición de que existen factores internos y externos a la organización que actúan como elementos causales del comportamiento de las variables de diseño organizativo y de sus procesos. ${ }^{2}$ Las organizaciones que alcancen un mejor

1 Entre algunas variables de diseño organizativo caben mencionar: centralización, formalización, especialización, ámbito de control, puestos de trabajo, sistema de incentivos, intensidad administrativa y procesos administrativos.

2 El diseño organizativo se refiere al conjunto de actividades, acciones y decisiones estratégicas y operativas que toman los directivos y gerentes con el propósito de definir los procesos, la estructura y las formas de organización que adopta la empresa en un momento determinado y bajo ciertas condiciones contextuales a las que tiene que adaptarse para poder alcanzar sus metas y objetivos (Zapata y Hernández, 2014). 
ajuste y adaptación con esos factores de contingencia, serán las más eficientes y tendrán por tanto una mayor probabilidad de supervivencia (Donaldson, 2001; Volberda, Van Der, Weerdt, Verwaal, Stienstra y Verdu., 2012; Akingbola, 2013; Kloviene, 2013), factores de contingencia tales como: el entorno, la estrategia, la tecnología, el marco institucional y el tamaño de la empresa. Sugiere así la teoría contingente, que el grado de percepción y comprensión de la incertidumbre del entorno tiene una relación directa con los resultados de la organización, con lo cual una alta percepción de incertidumbre de quienes la dirigen incrementa su atención sobre las estrategias, las estructuras y los procesos organizativos (Andrews, 2008; Heiens y Pleshko, 2011; Parnell, Lester, Long y Koseoglu., 2012; Ogunsiji y Akanbi, 2013).

Por otra parte, la teoría de dependencia de recursos sostiene que la organización no tiene la capacidad, ni es autosuficiente para producir y controlar todos los recursos y servicios que necesita para su crecimiento, mantenimiento y supervivencia. En consecuencia, debe recurrir a su entorno para lograr tales fines. Se observa entonces a la organización, como un modelo tradicional de sistema abierto dentro de un contexto social afectado por los factores del entorno que requiere de un continuo suministro de recursos para poder sobrevivir (Kazley y Ozcan, 2007; Schwalb et al., 2011), generándose con ello una dependencia externa limitada por la disponibilidad y la facilidad de acceso a esos recursos. Por esta razón, la organización tiene que adaptar sus acciones a las características del ambiente donde se desenvuelve, cuestión que pasa por implementar políticas y estrategias vinculadas con las relaciones de interdependencia con otras organizaciones (Pfeffer y Salancik, 19783; Ulrich y Barney, 1984; Wei, 2006; Nienhüser, 2008, Raza, Majeed, Maqsal y Mansoor, 2010; Peng y Beamish, 2014).

3 Pfeffer y Salancik (1978), proporcionaron las primeras referencias de la teoría de la dependencia de recursos. 
Se entiende por lo antes expuesto, que la necesidad de adquirir recursos produce la dependencia entre la organización y las unidades externas, con lo cual la teoría contingente y la dependencia de recursos mantienen una visión de adaptación, es decir, sostienen que los cambios en la organización, su comportamiento, sus procesosestructura-resultados y hasta su propia estabilidad, dependen y se explican a través de la influencia ejercida por los factores del entorno (Zapata y Mirabal, 2011).

Entre tanto, la perspectiva voluntarista, “[...] observa al individuo como la unidad básica de análisis y fuente de cambio organizacional" (Astley y Van de Ven, 1983, p. 247), recurriendo a su comprensión como elemento fundamental-explicativo de lo que ocurre dentro y alrededor de ella, considerándolo como un agente autónomo, pro-activo y auto-dirigido (Whittington, 1988). Así, Child $(1972,1997)$ como uno de los representantes de esta perspectiva teórica a través de su teoría la strategic choice, expone que la visión "determinista", refiriéndose a la perspectiva de control externo, es algo inadecuada debido a la poca atención que le ha prestado a la capacidad y poder de la coalición dominante para diseñar, organizar y dirigir a la empresa, y por tanto a su influencia para definir sus características y formas de organización que adopta.

La strategic choice, se define como un proceso esencialmente político por medio del cual quienes administran a la organización poseen el poder para decidir las acciones estratégicas y operativas, centrándose en el papel que desempeñan en la formación de las condiciones y los procesos dentro y alrededor de ella. Pero al mismo tiempo, con un sentido de equilibrio, la perspectiva de la strategic choice acepta que el entorno no es simplemente definido y seleccionado por la empresa, puesto que de aquel surgen fuerzas traducidas en amenazas u oportunidades constituidas en criterios o parámetros orientadores para llevar a cabo la acción y la elección estratégica (Child, 1997; Child, Leanne y Howard, 2003). De este modo, estos 
autores amplían el abanico de posibilidades a considerar cuando se trata de definir el futuro de la empresa sin perder de vista las circunstancias condicionadas por los factores que pueden limitar las acciones y las decisiones de los líderes de la organización.

Así pues, la presente investigación de carácter teórico tiene como propósito estudiar y evaluar diferentes tipologías del entorno organizacional y vincularlas con la percepción de directivos, gerentes y el diseño organizativo, considerando en su formación las perspectivas teóricas anteriormente consideradas. De esos análisis, surgen un conjunto de proposiciones que posteriormente podrán ser contrastadas en un estudio de carácter empírico, ya sea a través de metodologías cualitativas o cuantitativas.

Para alcanzar el propósito planteado, el presente trabajo ha sido estructurado en cinco apartados. El primero corresponde a esta introducción. Seguidamente, en el segundo apartado, se abordan los aspectos relacionados con la percepción del entorno y el significado de la incertidumbre que emana de él. En el tercero, se estudian diferentes tipologías del entorno basadas principalmente en trabajos seminales que desarrollan el tema, para posteriormente desarrollar en el cuarto apartado el análisis de los sesgos cognitivos y su relación con el proceso de percepción del entorno y sus características. Cabe destacar que en cada uno de esos apartados, se ofrecen un cuerpo de proposiciones que da lugar a las discusiones realizadas. Por último, se presenta un quinto apartado donde se exponen las reflexiones finales y las conclusiones.

\section{La percepción e interpretación del entorno y su incertidumbre}

La percepción e interpretación del entorno y su grado de incertidumbre, se ha convertido en un tema de estudio central en la literatura de la teoría de la organización, a si se observa en un buen número de investigaciones (Weick, 1979, 2005; Child, 1997; 
El entorno de la organización: un estudio de sus tipologías y su vinculación con la percepción directiva y el diseño organizativo

Pfeffer, y Salancik, 1978; Milliken, 1987; Walton y Dawson, 2001; Freel, 2005; Scott, 2010; López et al., 2011; Ogunsiji y Akanbi, 2013; Zabkar et al., 2013) entre otras. Constituyéndose en variables intermedias que enlazan el estudio del entorno con los procesos, la estructura organizativa y el desempeño organizacional. ${ }^{4}$

Child $(1972,1997)$, sostiene que el ejercicio de la actividad estratégica y el diseño organizativo, implican una evaluación previa del entorno por parte de la coalición dominante -directivos y gerentes-. La misma, se produce mediante la aplicación de filtros cognitivos durante el proceso de la percepción (Milliken, 1987; Child, 1997; Walton y Dawson, 2001; Mark, 2005; Zabkar, Cater, Bajde y Cater, 2013). Al respecto, Weick $(1969,1979)$ señala que las organizaciones sólo reconocen a su entorno mediante el proceso de percepción de quienes las dirigen, siendo producto de una ordenación abreviada, generalizada y corregible de las distintas experiencias dentro de la psiquis que sirven de marco de referencia inicial para la acción, transformados en mapas y sesgos cognitivos.

Frente a esas posiciones, se observa el entorno bajo una visión subjetiva y se entiende como un flujo continuo de información que puede ser filtrado, interpretado y definido en parte por los líderes de la organización (Whittington, 1988; Gopalakrishnan y Dugal, 1998; Freel, 2005 y Wong, et al., 2011). Es decir, la gerencia activamente selecciona o crea el entorno dónde quiere participar, competir y estructurar las decisiones necesarias para ajustar sus percepciones acerca del mundo que los rodea.

4 La percepción, es el proceso cognitivo por medio del cual el individuo capta lo que ocurre a su alrededor, identifica figuras, las formas de los seres humanos y las características del entorno, encajando lo observado con lo que esperaría ver en una situación similar. Con la percepción se interpreta y se entiende la información recibida a través de los sentidos, e implica la decodificación cerebral para encontrar algún significado a la información de forma tal que se pueda operar con ella o almacenarla (Banyard, Cassells, Green, Hartland, Hayes y Reddy, 1995). 
Bajo esa perspectiva, la organización se constituye en un proceso de interpretación individual, producto de la construcción y reconstrucción del ambiente percibido (Weick, 1969; Gopalakrishnan y Dugal, 1998 y Freel, 2005). Al respecto, Cameron (1986) indica que la efectividad de las organizaciones se corresponde en buen grado a una abstracción existente en las mentes de quienes las dirigen, lo cual da significado a las interpretaciones de sus resultados, pero que no necesariamente se corresponden a una realidad objetiva. De este modo, las organizaciones responden en parte a las percepciones subjetivas de sus líderes sobre las condiciones del entorno y en especial su incertidumbre, en lugar de considerar únicamente sus características objetivas tal como lo plantea el enfoque deterministacontingente ${ }^{5}$.

Así, la incertidumbre del entorno se constituye en uno de los principales problemas a enfrentar y resolver por la dirección de una organización, lo cual refleja una tendencia importante de la literatura que sostiene que cuanto mayor es la percepción de incertidumbre, los directivos y gerentes prestarán mayor atención a sus políticas y estrategias, y en general, a todos aquellos procesos y acciones que permitan mejorar las capacidades organizacionales (Agbejule, 2005; Andrews, 2008; Kanarattanavong y Ruenrom, 2009; López et al., 2011; Ogunsiji y Akanbi, 2013), siendo observado el entorno en términos de incertidumbre percibida. Entre esos procesos y acciones se encuentra la planificación flexible, que refleja la necesidad de procesos integradores de formación de la estrategia ante entornos dinámicos e inciertos (Andersen, 2004; Andersen y Nielsen, 2009).

5 Duncan $(1972,1973)$ en sus trabajos clásicos, señala que la incertidumbre está constituida por tres componentes básicos: (1) la carencia de información con respecto a los factores del entorno asociados con una situación de toma de decisión; (2) el desconocimiento del resultado de una decisión específica en los términos de cuanto perdería la organización si la decisión fuera incorrecta; y (3) la incapacidad de asignar cierta probabilidad éxito o fracaso sobre las decisiones. 
Por su parte Milliken (1987, p. 136), define la incertidumbre del entorno como "la incapacidad de un individuo de predecir algo (en el entorno de la organización) ${ }^{6}$ con exactitud, debido a que siente la carencia de información suficiente para pronosticar eventos o a la poca capacidad para discriminar entre datos relevantes e irrelevantes". El autor sugiere que la percepción de la incertidumbre sucede cuando los gerentes de la organización se percatan que ciertos cambios en el entorno no podrán ser pronosticados o manejados adecuadamente. Definición coincidente con la postura de Miles y Snow (1978, p. 195), quienes proponen que la percepción de la incertidumbre está determinada "por el reconocimiento del carácter imprevisible de las condiciones del entorno". Esto significa que los administradores al reconocerla, se ven motivados a tomar las decisiones que hagan posible enfrentarla poniendo en marcha los mecanismos necesarios para reducirla, absorberla o eliminarla (Jauch y Kraft, 1986; Kamps y Pólos, 1999; Jabnoun et al., 2003).

Asimismo, Milliken (1987) plantea que la percepción de la incertidumbre afecta la estrategia de la organización. En este sentido, propone que niveles altos de incertidumbre percibida están asociados con estrategias que buscan aumentar la diversificación dentro de una organización para disminuir su vulnerabilidad frente a las condiciones del entorno; es decir, se trata de identificar y desarrollar nuevos productos y servicios en un intento de reducir los efectos de un ambiente diversificado.

Tras el análisis del concepto de incertidumbre, vamos a revisar las aportaciones de diferentes trabajos clásicos que se enmarcan en las perspectivas de control externo, contingente y strategic choice.

En este sentido, Miles y Snow (1978) en su trabajo clásico expresan que dentro del enfoque de elección estratégica, el proceso de adaptación de la organización a las exigencias del entorno depende

6 Paréntesis nuestro. 
tanto de las condiciones objetivas impuestas por los factores contingentes (perspectiva contingente), como de las percepciones de los directivos y gerentes sobre esas condiciones (perspectiva voluntarista), las cuales finalmente contribuyen a definir los procesos de toma de decisiones estratégicas relacionados a la manera cómo debe ser organizada, diseñada y dirigida. En esa misma idea, Anderson y Paine (1975) concluyen que dependiendo de la percepción del entorno y de las propiedades internas de la organización y sus influencias asociadas, los gerentes tendrán una mayor o menor libertad en la elección de las estrategias necesarias para hacer frente a las distintas contingencias. Pero dejan claro que operando en altos niveles de incertidumbre $-O$ certidumbre - no necesariamente perciben el mismo grado de incertidumbre -o certidumbre-.

Entre tanto, Miles, Snow y Pfeffer (1974, p. 256) señalan que "en cada fase del proceso de ajuste organizativo y en ellas su variables de diseño, los gerentes tienen más o menos discrecionalidad para decidir y guiar a su organización a lo largo de diferentes cursos de acción". Esto significa, que el proceso cognitivo de la percepción del entorno guía de manera importante las decisiones estratégicas cuando se trata de alcanzar el ajuste más apropiado entre las variables internas de la organización y los factores del entorno. Por lo que la percepción contribuirá a la formación de la visión directiva vinculada con la forma cómo la empresa deberá ser organizada, dirigida, planificada, y el cómo las estrategias deben ser formuladas y puestas en marcha para que la organización responda adecuadamente tanto a las exigencias del entorno como a sus necesidades particulares (Peris, Peris-Ortiz y Zapata, 2006; Zapata y Canet, 2009).

De este modo, Miles et al. (1974) han caracterizado la percepción del entorno por parte de los gerentes en cuatro categorías principales: alta, media, poca y constante, cuyos grados de percepción son traducidos en diferentes respuestas organizativas y formas de organización. Dichos autores, intentan explicar cómo las distintas formas de organización se mueven a través de los procesos adaptativos y desarrollan pautas de comportamiento que permiten su 
adaptación. Al modelo lo denominan ciclo adaptativo, y responde a un proceso de ajuste dinámico y complejo donde la empresa tendrá que resolver tres tipos de problemas: empresarial, de ingeniería y administrativo. Conceptualizan en dicho ciclo tres elementos fundamentales en el proceso del diseño y desarrollo organizacional: la acción, la elección y la adaptación, notando que el mismo puede ser iniciado en cualquiera de ellos.

Vemos pues que en el modelo de Miles y Snow subyace la perspectiva de control externo, al dejar claro la capacidad adaptativa de la organización, pero asimismo, no dejan de lado, como sí lo hace el modelo contingente tradicional, la participación de la dirección en las distintas fases del ciclo adaptativo y por consiguiente en los destinos y comprensión de los problemas organizacionales, o sea con una visión también próxima a lo establecido por la teoría de la strategic choice dentro del marco de la perspectiva voluntarista.

En relación con esta perspectiva, el entorno debe ser percibido por los directivos, y en función de esa percepción actuarán. En ese sentido, Daft y Weick (1984, p. 286) expresan que "la interpretación es el proceso mediante el cual se traducen los eventos del entorno tomado en cuenta por los gerentes, se desarrollan los modelos para entenderlos y revelar sus resultados, y se ensamblan los esquemas conceptuales entre ellos (se refieren a los mapas cognitivos $)^{7}$ ". El concepto lo organizan en tres fases: exploración -scanning-, interpretación -interpretation- y aprendizaje -learning-.

La fase de exploración, es el monitoreo, búsqueda y obtención de datos con el propósito de identificar las tendencias clave, los cambios y los eventos del entorno que en un momento dado pueden afectar el funcionamiento y desempeño de la empresa (Daft y Weick, 1984; Daft, Sormunen y Parks, 1988; Sawyerr, Ebrahimi y Thibodeaux, 2000; Albright, 2004). De otro modo, la organización experimenta la fase de interpretación, cuando son introducidos nuevos constructos

\footnotetext{
Paréntesis nuestro.
} 
en los mapas cognitivos colectivos de la organización y se comparten percepciones entre sus miembros (Daft y Weick, 1984). Esta implica la identificación de amenazas y oportunidades del entorno, "requiriéndose de los gerentes la evaluación previa del significado e importancia de cada cambio, evento y tendencia observada y atendida durante la fase de exploración" (Milliken, 1990, p. 44). Y, finalmente, la tercera fase del proceso de interpretación propuesto por Daft y Weick (1984), es la de aprendiraje que implica:

a) Una nueva respuesta o acción basada en la fase de interpretación; y

b) La adquisición de nuevos datos y desarrollo de conocimientos basado en los resultados de las acciones llevadas a cabo a través de las relaciones entre la organización y su entorno, generándose de esa acción un feedback para las fases de interpretación y exploración.

Destacan, además, que la fase de aprendizaje puede verse afectada por factores tales como: las creencias, la formación, la cultura y las percepciones de los directivos y gerentes, y además, por los objetivos, las metas y las políticas de la organización.

En la figura N. $^{\circ} 1$, se ilustra las tres fases del proceso de interpretación descrito en el párrafo anterior.

Figura N. ${ }^{\circ} 1$

Relación entre las fases de exploración, interpretación y aprendizaje de la organización

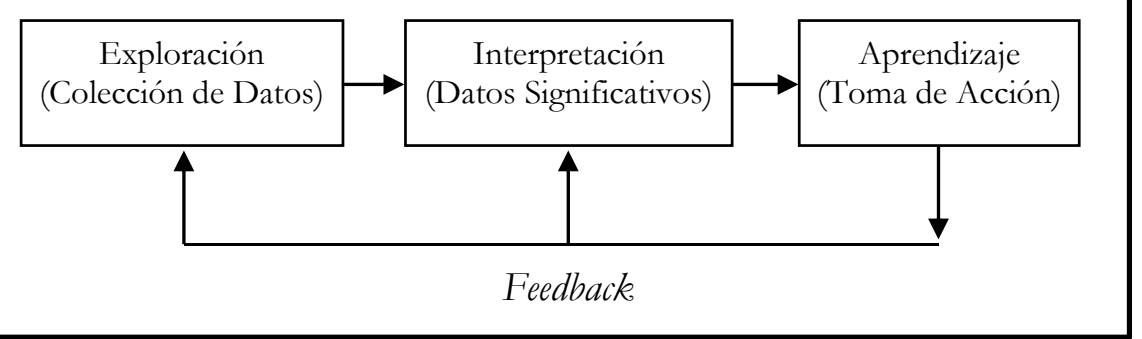

Fuente: Daft y Weick (1984). 
Por su parte, Galbraith $(1973,1977)$ ofrece un modelo dentro del marco de la teoría contingente, que concibe a la organización como una red de procesamiento de información basado en dos ideas esenciales:

1. La incertidumbre del entorno puede afectar el trabajo realizado en la organización. Así "mientras mayor es la incertidumbre sobre las tareas o el trabajo, mayor será la cantidad de información que deberá ser procesada por los decisores durante la ejecución de su trabajo para poder alcanzar un nivel adecuado de actuación” (Galbraith, 1973, p. 4, 1977, p. 36). De esta forma, sostiene que si las tareas son entendidas antes de que se lleven a cabo, entonces pueden ser programadas con anticipación y desarrolladas con facilidad; y cuando es al contrario, durante la realización de las tareas se irá adquiriendo los conocimientos, las habilidades y los recursos necesarios para ejecutarlas. En otras palabras, un procesamiento emergente de información; y

2. Bajo incertidumbre de tareas, la organización empleará distintas estrategias de procesamiento de información, la cual pueden ser:

a) Reducir la necesidad de procesamiento de la misma a través de la creación o consumo de recursos holgados - por ejemplo, aumentando el tiempo programado de producción, las horas hombres invertidas y los plazos de entrega de los productos-, o creando tareas autónomas mediante la asignación a cada grupo de trabajo de los recursos que requiere; $\mathrm{y}$

b) Aumentar la capacidad de procesamiento de información por medio de la inversión en sistemas de información vertical -tecnologías, programas de almacenamiento, formalización de los canales de comunicación, etc.-, o la creación de procesos de toma de decisión conjunta -contacto directo, roles y grupos de enlace, rol integrador, gerente integrador y diseño en forma de matriz-. 
La importancia de los trabajos de Galbraith $(1973,1977)$, es que tiende otro puente teórico importante para la compresión del efecto de la incertidumbre del entorno sobre las características del trabajo y el diseño de la organización.

En resumen, se puede plantear que el grado de incertidumbre no es una característica constante en una organización puesto que depende fundamentalmente de la capacidad de percepción de sus miembros. Algunos individuos tienen mayor tolerancia a la ambigüedad o a la complejidad, y por consiguiente perciben situaciones con menos incertidumbre en relación a otros individuos. En esto, pueden intervenir múltiples factores tales como la experiencia del directivo o gerente, su posición frente a la jerarquía o cargo ocupado, el grado de formación, la disposición al cambio, la actitud para enfrentarse a la incertidumbre, los valores, las creencias y los sesgos cognitivos que influyen en el proceso de toma de decisiones. Con lo cual, el aspecto clave es que el grado de incertidumbre percibida no sólo depende de las características del entorno y de sus exigencias, sino que también de las diferencias existentes entre directivos y gerentes. Evidentemente esto tendrá un impacto importante en las decisiones tomadas por los responsables de dirigir a la organización.

Finalmente, de acuerdo a los aspectos desarrollados en los párrafos anteriores se ofrecen a continuación las siguientes proposiciones generales:

P1: Existe una relación significativa entre el grado de percepción de la incertidumbre del entorno por parte de directivos y gerentes, y la naturaleza y características de las políticas y estrategias que se deben tomar en la organización.

En particular:

P1.1: Cuanto mayor es el grado de incertidumbre percibida, mayor racionalidad en los procesos y estrategias adoptados. 
P1.2: Cuanto mayor es el grado de incertidumbre percibida, las estrategias se orientan al mantenimiento y mejora de las capacidades organizacionales, a través, entre otras, de las políticas de capacitación y desarrollo.

P2: Bajo las mismas condiciones del entorno y en organizaciones de la misma naturaleza y fines, no todos los directivos y gerentes tienen la misma percepción de la incertidumbre del entorno.

P3: La capacidad de percepción de las características del entorno por directivos y gerentes, contribuirá en la definición de los diseños organizativos.

\section{Tipologías de entorno y la incertidumbre percibida}

En su trabajo seminal, Thompson (1967) concluye que el entorno percibido está constituido por dos dimensiones: homogéneo/ heterogéneo y estable/dinámico, las cuales determinarán de manera importante cómo deberá ser definida la estructura organizativa. La dimensión homogéneo/ heterogéneo, se refiere al grado de variedad o diversificación del entorno en distintos grupos que afectan a la organización -proveedores, clientes, competencia, órganos de gobierno-; es decir, con características distintivas de diferenciación. Por otra parte, la dimensión estable/dinámico está relacionada con el carácter cambiante del entorno en cuanto a los aspectos tecnológicos, el marco normativo, la posición de los competidores, el índice de aparición o desaparición de empresas en los mercados y los cambios en los productos y servicios ofrecidos por la organización o demandados por el entorno.

Por su parte, Duncan (1972) en su investigación seminal bajo el marco de la teoría contingente y sus posteriores trabajos de 1973 y 1979, enfatiza en el hecho de que las respuestas de la organización se ven influidas por la percepción de la gerencia sobre la incertidumbre del entorno. De esta manera, señala que en una situación de incertidumbre, el individuo se siente incapacitado tanto para asignar con 
exactitud la probabilidad que ocurra o cambie algún evento en el futuro, como para predecir las consecuencias de una decisión.

$\mathrm{El}$ autor, define cuatro niveles de percepción de la incertidumbre: baja, moderadamente baja, moderadamente alta y alta incertidumbre, que interaccionan con las dos dimensiones del entorno identificadas por el autor:

1. Cambio del entorno -estático y dinámico-; y

2. Complejidad del entorno-simple y complejo ${ }^{8}$.

La combinación de esas dos dimensiones produce cuatro circunstancias recogidas en la tabla $\mathrm{N} .^{\circ} 1$.

\section{Tabla N. ${ }^{\circ} 1$}

Dimensiones del entorno e incertidumbre percibida

\begin{tabular}{|c|c|c|}
\hline Entorno & Simple & Complejo \\
\hline \multirow[b]{2}{*}{ Estático } & Baja percepción de incertidumbre & $\begin{array}{l}\text { Moderadamente baja percepción } \\
\text { de incertidumbre }\end{array}$ \\
\hline & $\begin{array}{l}\text { - Número pequeño de factores y } \\
\text { componentes del entorno. } \\
\text { - Los factores y componentes son } \\
\text { algo similares. } \\
\text { - Los factores y componentes se } \\
\text { mantienen básicamente iguales y } \\
\text { no son cambiantes. }\end{array}$ & $\begin{array}{l}\text { - Gran número de factores y com- } \\
\text { ponentes del entorno. } \\
\text { - Los factores y componentes no son } \\
\text { similares unos respecto de otros. } \\
\text { - Los factores y componentes se } \\
\text { mantienen básicamente iguales y } \\
\text { no son cambiantes iguales. }\end{array}$ \\
\hline \multirow[b]{2}{*}{ Dinámico } & $\begin{array}{l}\text { Moderadamente alta percepción de } \\
\text { incertidumbre }\end{array}$ & Alta percepción de incertidumbre \\
\hline & $\begin{array}{l}\text { - Número pequeño de componentes } \\
\text { y factores en el entorno. } \\
\text { - Los factores y componentes son } \\
\text { algo similares. } \\
\text { - Los factores y componentes están } \\
\text { en un proceso continuo de cambios. }\end{array}$ & $\begin{array}{l}\text { - Gran número de factores y com- } \\
\text { ponentes del entorno. } \\
\text { - Los factores y componentes no } \\
\text { son similares uno de otros. } \\
\text { - Los factores y componentes están } \\
\text { en un proceso continuo de cambios. }\end{array}$ \\
\hline
\end{tabular}

Fuente: adaptado de Duncan $(1972,1973,1979)$.

8 Duncan (1972, p. 325) define la dimensión simple-compleja, como el número de factores y/o componentes tomados en consideración en la toma de decisiones y su similitud. Por otra parte, la dimensión estática-dinámica es el grado en el cual esos factores se mantienen básicamente iguales en el tiempo, o están en continuo proceso de cambio. 
En la misma línea de Duncan, Mintzberg (1979) sugirió cuatro tipos básicos de entornos derivados de la combinación de las dimensiones estable-dinámica y simple-compleja. Según el autor, el dinamismo del entorno está determinado por una variedad de factores, incluidos, por ejemplo, los gobiernos inestables, los cambios no previstos en la economía, las variaciones en las demandas de los clientes, una tecnología o base de conocimiento en rápida transformación, los cambios creados en los propios talleres de la empresa o en el tamaño de la organización. El entorno dinámico hace que el trabajo resulte incierto e impredecible; es decir, afecta la variable predictibilidad del trabajo. Por otra parte, la complejidad del entorno se relaciona con la comprensibilidad del trabajo. En ese sentido, el entorno es complejo en la medida que la organización requiere de una gran cantidad de conocimientos sofisticados sobre los productos, clientes, proveedores y otros componentes de ese ambiente. Se convierte en sencillo, cuando el conocimiento puede racionalmente descomponerse en elementos fáciles de comprender, por ejemplo el conocimiento acumulado, registrado y sistematizado.

Pero la idea de Mintzberg (1979) era ofrecer diferentes características de las organizaciones bajo determinadas condiciones del entorno. A continuación, se presentan en la tabla N. ${ }^{\circ} 2$.

\section{Tabla N. ${ }^{\circ} 2$}

\section{Entornos básicos de la organización}

\begin{tabular}{|c|l|l|}
\hline Entorno & \multicolumn{1}{|c|}{ Estable } & \multicolumn{1}{c|}{ Dinámico } \\
\hline Simple & $\begin{array}{l}\text { Centralizada- burocrática } \\
\text { Coordinación: normalización de } \\
\text { los procesos de trabajo }\end{array}$ & $\begin{array}{l}\text { Centralizada-orgánica } \\
\text { Coordinación: supervisión directa }\end{array}$ \\
\hline Complejo & $\begin{array}{l}\text { Descentralizada-burocrática } \\
\text { Coordinación: normalización de } \\
\text { las habilidades }\end{array}$ & $\begin{array}{l}\text { Descentralizada-orgánica } \\
\text { Coordinación: adaptación mutua }\end{array}$ \\
\hline
\end{tabular}

Fuente: Mintzberg (1979). 
Es de destacar que los conceptos de Duncan $(1972,1973)$ y Mintzberg (1979) en relación a la dimensión simple-compleja parecieran ser explicado como puntos de vista diferentes pero realmente tienen coincidencia. Mientras que el primero de los autores habla de la complejidad en términos de cantidad de factores existentes en el entorno, Mintzberg la asocia con el grado de dificultad de los conocimientos requeridos para elaborar los productos exigidos por el entorno. Es decir, mientras más difíciles y complejas son las actividades necesarias para la producción de bienes o servicios, más conocimientos sofisticados serán necesarios y mayor la complejidad. Ahora bien, el punto de coincidencia entre los conceptos de ambos autores viene dado por el hecho de que en la medida que sea necesario considerar una mayor cantidad de factores del entorno para tomar decisiones -posición de Duncan-, en esa medida se producirá una mayor cantidad de elementos de conocimientos, más o menos complejos, para comprender y analizar todos esos factores y poder así dar las respuestas adecuadas. En el fondo, ambas posiciones tratan el problema de obtener los conocimientos necesarios para entender y llevar a cabo el trabajo más o menos complejo, de acuerdo a las demandas y a los cambios exigidos del entorno.

Dess y Beard, (1984) observan al entorno como una fuente de información y recursos para la organización, y Harrington, Lema, Reed y Kendall (2004) como un enlace entre la formulación estratégica y el desempeño. Estos autores definieron tres dimensiones del entorno:

1. La complejidad: relacionada con la hetereogenidead-homogeneidad de los factores del entorno, la cual se inserta dentro de la dimensión simple-compleja.

2. El dinamismo: vinculada con la estabilidad-inestabilidad, siendo la incertidumbre un componente importante y se puede ubicar dentro del esquema de entorno dinámico-estable. 
3. La munificencia, tiene que ver con la dependencia de la empresa con los recursos que requiere del entorno, es decir, esto último asociado básicamente con la teoría de la dependencia de recursos.

Lo importante es reflexionar si las dos principales dimensiones (simple-compleja y estable-dinámica), tienen el mismo impacto sobre la incertidumbre. Con lo cual, se puede pensar que en entornos dinámicos los factores considerados para la toma de decisiones estratégicas para la organización están en permanente cambio y transformación, circunstancias que generan nuevas situaciones y desconocimiento e ignorancia en su manejo adecuado. En consecuencia, los miembros de la organización deberán someterse a procesos permanente de aprendizaje-readaptación y búsqueda de nuevos conocimientos para poder llevar a cabo las tareas que permitan satisfacer las exigencias de cambios o variedad del entorno.

De esta manera, se puede pensar que las unidades de decisión estratégicas en entornos dinámicos experimentan significativamente mayor incertidumbre en la toma de decisiones con relación a aquellas desenvueltas en entornos complejos pero estables, con lo cual las diferencias en la percepción de la incertidumbre entre las unidades con entornos complejos no son tan significativas al menos que ese medio sea también dinámico.

Lo anterior, se vincula a la forma de abordar el tema de la percepción por Weick (1969), cuando hace referencia a la ley de la variedad necesaria afirmando que "la variedad de un sistema debe ser, al menos, tan grande como la variedad del entorno al que trata de ajustarse. Es decir, sólo la variedad puede regular la variedad" (Weick, 1969, p. 189)9. Trasladada esa definición a la realidad de

\footnotetext{
9 La ley de variedad, se corresponde al pensamiento de Lawrence y Lorsch (1967a, 1967b) en el sentido que mientras más complejidad exista en el sector donde participa la organización, mayor será la necesidad de diferenciación organizativa-vertical, horizontal y espacial- para poder enfrentar los distintos aspectos de esa complejidad.
} 
las organizaciones, significa que los procesos organizativos aplicados a entradas variadas deben ser en sí variadas, es el punto en el que la variedad de los procesos debe igualar a la variedad de las entradas. Idea ésta consistente con el pensamiento de Lawrence y Lorsch (1967a, 1967b) y March y Simon (1958). Los primeros, cuando proponen que una de las razones principales de la diferenciación de la organización en distintos subsistemas es que posee la capacidad suficiente para manejar con eficiencia diferentes subentornos. Los segundos, cuando hacen referencia a la racionalidad limitada y a los procesos de simplificación cognitiva, indicando que una de las técnicas fundamentales para simplificar un problema dentro de la organización y enfrentar la racionalidad limitada, es "fraccionar el problema en un número de partes casi independiente, de forma tal que cada unidad de la organización maneje una de esas partes y pueda omitir las otras en su definición de la situación" (March y Simon, 1958, p. 67). Este razonamiento sugiere que los directivos suelen enfrentarse a entornos complejos intentando hacerlos sencillos; sin embargo, cuando la complejidad proviene del desarrollo de la propia tarea en sí misma, en el sentido en que Perrow (1970) define la complejidad, esta pueden ser más difícil de reducir. Y si esta actividad compleja está relacionada, por ejemplo, con la innovación, y por tanto se asocia a un entorno dinámico, es probable que el nivel de incertidumbre percibida sea aún mayor.

De lo expuesto anteriormente, se plantean las siguientes proposiciones:

P4: Existe una relación positiva y significativa entre la percepción de incertidumbre del entorno de los directivos o gerentes y el grado de dinamismo del entorno.

P4.1: La percepción de incertidumbre será mayor si estamos en un entorno dinámico y complejo a la vez.

P5: La dimensión estática-dinámica del entorno contribuye más al grado de incertidumbre que la dimensión sencilla-compleja. 


\section{Los sesgos cognitivos y la percepción del entorno}

La percepción del entorno está asociada en parte, por la influencia que sobre el comportamiento de los directivos y gerentes tienen ciertas convicciones o sesgos cognitivos, siendo su estudio un tema central de la teoría de la organización (Schwenk, 1986, 1988; Keil, Gordon y Rai, 2007; Zapata y Canet, 2009; Pissaris, Weinstein $y$ Stephan, 2010; Gudmundsson y Lechner; 2013; Reitzig y Sorenson, 2013). Por ejemplo, los compromisos con el pasado hacen que las cosas se hagan en la organización de acuerdo a sus creencias, tradiciones, costumbres, rituales y supersticiones. "Muchas de las creencias del éxito del pasado se atrincheran en las estructuras administrativas y directivas [...], y son a veces muy difíciles de cambiar" (Pfeffer y Salancik, 1978, p. 82). Las organizaciones bajo el compromiso de hacer las cosas como siempre las han hecho, quedan sujetas a una manera fija de seguirlas haciendo y como consecuencia de ello pueden caer en el riesgo de no darse cuenta de los cambios y de las nuevas exigencias de su contexto externo (Mintzberg, Ahlstrand y Lampel, 1998).

Cabe destacar aquí los trabajos seminales sobre psicología cognitiva de Neisser $(1976,1981)$, quien señala que las estructuras mentales o cognitivas representan un marco de referencia espacial, temporal y conceptual inespecífica, pero armonizada, de las experiencias previas del individuo. El autor indica que las estructuras cognitivas fundamentales para generar la visión de los individuos son los esquemas anticipatorios. Estos, forman el medio a través del cual los individuos se preparan para recibir cierto tipo de información y por tanto, permiten controlar las actividades percibidas a través de los diferentes sentidos. "Es, en alguna medida, una manera que el pasado afecte al futuro y en consecuencia la información ya adquirida establece, en gran parte, la información que se recibirá después". Así, "el esquema determina lo percibido puesto que la información únicamente puede ser extraída si hay un formato desarrollado dispuesto a recibirla" (Neisser, 1981, p. 41); en otras 
palabras, le sirve al individuo de guía para seleccionar lo recibido de su contexto. El esquema, aclara el autor, no constituye un elemento estático o invariable y en esa idea expresa: "el esquema es interno al individuo, modificable por la experiencia y de algún modo concreto con respecto a lo que percibe" (Neisser, 1981, p. 67). Pero señala a su vez, que aunque las estructuras y los sesgos cognitivos hacen posible el recuerdo, también tiene algunos efectos negativos en el sentido que tiende a introducir prejuicios y distorsiones en la construcción inicial de los mapas cognitivos, y por tanto en la toma de decisiones.

Los investigadores en psicología cognitiva han identificado una serie de sesgos cognitivos a los cuales están sujetos los individuos en sus juicios o acciones (Tversky y Kahneman, 1973, 1974; Makridakis, 1990; Fisk, 2002; Castañeda et al., 2003; Keil et al., 2007; Pissaris, et al., 2010; Gudmundsson y Lechner; 2013), siendo éstos reglas de decisión, mecanismos cognitivos y opiniones subjetivas que las personas utilizan para ayudarse en el proceso de toma de decisiones (Busenitz y Barney, 1997, 1999; Arnott, 2006; Keil et al., 2007; Gudmundsson y Lechner; 2013; Reitzig y Sorenson, 2013), y que les permiten desarrollar modelos simplificados del mundo o de la realidad que los rodea con el propósito de facilitar las propuestas de solución a los problemas de diseño organizativo (Zapata y Canet, 2009). En otras palabras, los encargados de tomar decisiones estratégicas dependen de algunas reglas de juicio o sesgos cognitivos para simplificar las situaciones complejas, y así poder establecer los modelos de decisiones y acciones que determinan el curso de acción de la organización.

Schwenk $(1986,1988)$, en sus trabajos relacionados con los procesos de simplificación cognitiva y la toma de decisiones estratégicas, muestra que algunos sesgos cognitivos pueden interactuar y reforzarse entre ellos. Por ejemplo, indica que los gerentes en su actividad de toma de decisiones están sometidos a una serie de sesgos cognitivos, entre ellos al sesgo de ilusión de control. En presencia de dicho sesgo, los gerentes tienden a buscar información -sesgo 
de disponibilidad- para confirmar y apoyar esa ilusión; es decir, se fortalece, promueve o aumenta la percepción de control sobre alguna situación o evento determinado. También, por ejemplo, es posible que el razonamiento por analogía pueda ser reforzado por el sesgo de ilusión de control, en el caso que una empresa decida adquirir un negocio relacionado (Duhaime y Schwenk, 1985). En otras palabras, se toma una decisión en función de procesos ya conocidos en relación a otros -analogía-, y el individuo se concentra y evalúa, por simplificación, sólo aquellos factores que domina $\mathrm{o}$ cree dominar -ilusión de control-, dejando de lado otros factores que podrían cambiar la decisión. Igualmente, la disponibilidad de información -sesgo de disponibilidad-puede servir de apoyo o de estímulo a un sesgo de anclaje -la influencia de la información inicial sobre la decisión-, en el juicio o decisión estratégica a adoptar por los gerentes (Schoemaker, 1993, Schwenk, 1986).

Gudmundsson y Lechner (2013) concluyen que los sesgos cognitivos mantienen una relación estrecha con la supervivencia de la empresa, teniendo el sesgo de exceso de confianza su principal influencia negativa, y sus efectos se magnifican cuando se combina con el sesgo de optimismo poco realista y el de la desconfianza, describiendo dos tipos cognitivos distintivos de empresarios asociados a la no-supervivencia. Manifiestan los autores que los empresarios con exceso de confianza son propensos a pensar demasiados en sus capacidades y por tanto tienen una mayor probabilidad de cometer errores en la toma de decisiones ${ }^{10}$.

De esa misma manera, y bajo la idea de la escuela cognitiva del pensamiento estratégico, Mintzberg et al. (1998) señala que los investigadores se han interesado en el modo cómo los individuos

10 Gudmundsson y Lechner (2013, p. 290) expresan que "si no fuera por los sesgos cognitivos, la creación de empresas probablemente no se llevaría a cabo con la frecuencia con la que se observa”, esto seguramente con independencia de que si fracasan o tienen éxito. 
simplifican y procesan la información, interviniendo en ese proceso un conjunto de sesgos de criterios con consecuencias importantes en las decisiones tomadas en la organización, tales como la formulación e implantación de las estrategias empresariales y aquellas vinculadas con el diseño organizativo. En ese sentido, Weick (1995) estudia cómo las personas reducen la ambigüedad y la incertidumbre a través de significados compartidos ya sean individuales o colectivos, fundamentados en sus valores, reglas y criterios de juicios (sesgos cognitivos), preferencias e interpretaciones del contexto que los rodea. Esto implica el desarrollo de un curso de acción retrospectivo de imágenes que permiten a las personas racionalizar lo que piensan y hacen en las organizaciones (Weick, Sutcliffe y Obstfeld, 2005). La idea de que la forma de trabajar del pasado tiene un impacto determinante en la manera de trabajar del presente, está vinculada a la tendencia de los individuos a resolver los problemas en función a sus experiencias pasadas -sesgos de percepción selectiva y de tendencia conservadora-, con la información inicial que poseen -sesgo cognitivo de anclaje-, y con poca probabilidad de cambiar de idea -resistencia al cambiofrente a nuevas situaciones.

Lo anterior puede estar relacionado con la interpretación incorrecta de las demandas del entorno, que ocurre cuando la organización no percibe de manera adecuada las presiones o cuestionamientos que se le hacen desde su entorno sobre la calidad de sus salidas- bienes o servicios-. Cuestión que Pfeffer y Salancik (1978) vinculan con los procesos de percepción y filtros de información aplicados por los individuos cuando tratan de definir su entorno -enact environment-; es decir, los individuos se concentran fundamentalmente en lo que están preparados para atender y sobre aquellos aspectos relevantes para su trabajo, situación que los puede llevar a no considerar información de interés o relevante para la organización como un todo, y a perder su capacidad tanto para equilibrar las demandas de los diferentes grupos externos que requieren de sus bienes o servicios, como para identificar sus propias prioridades. 
En este sentido, y basándonos en los aspectos discutidos en este apartado se pueden formular las siguientes proposiciones generales:

P6: Los sesgos cognitivos de los directivos y gerentes tienen una influencia significativa en su capacidad para interpretar las características y naturaleza del entorno.

P7: Existe una relación significativa entre los sesgos cognitivos y el comportamiento de los directivos y gerentes acerca de la manera como debe ser diseñada, administrada y dirigida la organización.

\section{Conclusiones}

Los niveles de percepción e interpretación de las condiciones y características del entorno del entorno (estable/dinámico-simple/complejo) por parte de la dirección de la empresa, contribuirá a la formación de su visión de cómo debe ser diseñada, dirigida y planificada, y cuáles deben ser las estrategias formuladas y puestas en marcha para responder adecuadamente tanto a las circunstancias del entorno como a sus necesidades particulares. En otras palabras, cobra relevancia el concepto de visión subjetiva del entorno.

Esas percepciones sirven de enlace entre las características objetivas del entorno y las distintas acciones llevadas a cabo en la organización -individual o colectiva-, que pueden generar diferentes respuestas, puesto que las personas no perciben y evalúan el entorno de la misma forma, sino que hay factores individuales que así lo determinan. Factores, por ejemplo, como la experiencia, la formación, los valores y las creencias, o simplemente los sesgos cognitivos de cada individuo. Es decir, bajo iguales condiciones y características del entorno, los directivos y gerentes de las mismas o de distintas organizaciones percibirán la incertidumbre en grados diferentes. Es por ello que las empresas, operando en un mismo sector y circunstancias contextuales muy parecidas, son diseñadas y dirigidas con políticas y estrategias a veces bastante disímiles. 
Del mismo modo, en la medida que la capacidad de percepción del gerente o directivo de las condiciones y características del entorno mejore, en esa misma medida podrá comprender y percibir en mayor grado su complejidad y ambigüedad, así como la incertidumbre que emana de él y por tanto de su propia naturaleza, características y tipología. Debido a eso, posiblemente estará en mejores condiciones para tomar las decisiones y dar las respuestas más adecuadas y oportunas para el adecuado curso de acción de la organización.

Indudablemente, en todas estas circunstancias descritas juegan un papel de vital importancia los procesos simplificadores del pensamiento y predisposiciones mentales tales como los mapas y sesgos cognitivos que influyen en el comportamiento del individuo y por ende en la toma de sus decisiones. Así, el reconocimiento de la incertidumbre del entorno no sólo responde a la consideración de los factores externos de quienes dirigen y administran a la organización, tal como lo argumenta el modelo de control externo de la organización, sino también a las características personales y cognitivas del individuo como lo plantea la perspectiva voluntarista. Por tanto, puede considerarse que la disponibilidad de recursos y capacidades de la organización sea producto, en parte, de una acción subjetiva percibida e interpretada de las condiciones y características del entorno.

\section{Referencias bibliográficas}

Agbejule, A. (2005). The Relationship Between Management Accounting Systems and Perceived Environmental Uncertainty on Managerial. Accounting and Business Research, 23(4), 295-305.

Albright, K. (2004). Environmental Scanning: Radar For Success. Information Management Journal, 38(3), 38-45.

Akingbola, K. (2013). Contingency, fit and flexibility of HRM in Nonprofit Organizations. Employee Relations, 25(5), 479-49. 
Andersen, T. (2004). Integrating Decentralized Strategy Making and Strategic Planning Processes in Dynamic Environments. Journal of Management Studies, 41, 1271-1299.

Andersen, T. y Nielsen, B. (2009). Adaptive strategy making: The effects of emergent and intended strategy modes. European Management Review, 6(2), 94-106.

Anderson, C. y Paine, F. (1975). Managerial Perspective and Strategic Behavior. Academy of Management Journal, 18(4), 811-823.

Andrews, R. (2008). Perceived Environmental Uncertainty in Public Organizations An Empirical Exploration. Public Performance and Management Review, 32(1), 25-50.

Astley G. \& Van de Ven A. (1983). Central Perspective and Debates in Organizations Theory. Administrative Science Quarterly, 28(2), 245-273.

Arnott, D. (2006). Cognitive Biases and Decision Support Systems Development: A Design Science Approach. Information Systems Journal, 16, 55-78.

Banyard, P., Cassells, A., Green P., Hartland, J.; Hayes, N. \& Reddy, P. (1995). Introducción a los procesos cognitivos. Barcelona: Ariel Psicología.

Busenitz, L. \& Barney, J. (1997). Differences Between Entrepreneurs and Managers in Large Organizations: Biases and Heuristics in Strategic Decision-Making. Journal of Business Venturing, 12, 9-30.

Busenitz, L. (1999). Entrepreneurial Risk and Strategic Decision Making: It's a Matter of Perspective. The Journal of Applied Behavioural Science, 35(3), 325-338.

Cameron K. S (1986). A Study of Organizational Effectiveness and its Predictors. Management Science, 32, 86-112. 
Child, J. (1972). Organization Structure, Environment and Performance: The Role of Strategic Choice. Sociology, 6, 1-22.

Child, J. (1997). Strategic Choice in the Analysis of Action, Structure, Organizations and Environment, Organization Studies, 18, 43-76.

Child, J.; Leanne, Ch. \& Howard, D. (2003). The Performance of Cross-Border Units in China: A Test of Natural Selection, Strategic Choice and Contingency Theories. Journal of International Business Studies, 43(3), 242-254.

Castañeda, J.; Sánchez, C.; Quintana, D. \& González, T. (2003). Razonamiento Predictivo y Contenidos Sesgados: Un Estudio con Personas de Hábitat Rural y Urbano. Cognitiva, Volumen, 15(1), 67-82.

Daft, R. \& Weick, K. (1984). Toward a Model of Organizations as Interpretation Systems. Academy of Management Review, 9(2), 284-291.

Daft, R., Sormunen, J. \& Parks, D. (1988). Chief Executive Scanning, Environmental Characteristics, and Performance Company: An Empirical Study. Strategic Management Journal, 9(2), 123-139.

Dess, G. \& Beard D. (1984). Dimensions of Organizational Task Environments. Administrative Science Quarterly, 29, 52-73.

Dill, W. (1958). Environment as an Influence on Managerial Autonomy. Administrative Science Quarterly, 20, 409-443.

Donaldson, L. (2001). The Contingency Theory of Organizations. California: Sage Publications.

Duhaime, I. \& SchwenK, Ch. (1985). Conjectures on Cognitive Simplification in Acquisition and Divestment Decision Making. Academy of Management Review, 10(2), 287-295. 
Duncan, R. (1972). Characteristics of Organizational Environments and Perceived Environmental Uncertainty. Administrative Science Quarterly, 17(3), 313-327.

Duncan, R. (1973). Multiple Decisions Making Structure in Adapting to Environmental Uncertainty. Human Relations, 26, 273-291.

Duncan, R. (1979). What Is the Right Organization Structure? Organizational Dynamics, 7(3), 59-80.

Fisk, J. (2002). Judgments Under Uncertainty: Representativeness or Potential Surprise? British Journal of Psychology, 93(4), 431449.

Freel, M. (2005). Perceived Environmental Uncertainty and Innovation in Small Firms. Small Business Economics, 25(1), 49-64.

Galbraith, J. (1973). Designing Complex Organizations. Reading, Massachusetts: Addison-Wesley.

Galbraith, J. (1977). Organization Design. New Jersey: AddisonWesley.

Gopalakrishnan, Sh. \& Dugal, M. (1998). Strategic Choice Versus Environmental Determinism: A Debate Revisited. International Journal of Organizational Analysis, 6(2), 146-164.

Gudmundsson, S. y Lechne, Ch. (2013). Cognitive Biases, Organization, and Entrepreneurial Firm Survival. European Management Journal, 31, 278-294.

Harrington, R.; Lemak, D.; Reed, R. y Kendall, K. (2004). A Question of Fit: The Links Among Environment, Strategy Formulation and Performance. Journal of Business Management, 10(1), 15-38. 
Heiens, R. \& Pleshko, L. (2011). A Contingency Theory Approach to Market Orientation and Related Marketing Strategy Concepts: Does Fit Relate to Profit Performance. Managementy Marketing, 6(1), 19-34.

Jabnoun, N.; Khalifah, A. y Yusuf, A, (2003). Environmental Uncertainty, Strategic Orientation, and Quality Management: Contingency Model. The Quality Management Journal, 14(4), 17-30.

Jauch, L. \& Kraft, K. (1986). Strategic Management of Uncertainty. Academy of Management Review, 11(4), 777-790.

Khalifa, M.; Yan-Yu, A. \& Ning, K. (2008). Knowledge management systems success: a contingency perspective. Journal of Knowledge Management, 8(1), 119-132.

Kamps, J. \& Pólos, L. (1999). Reducing Uncertainty: A Formal Theory of Organizations in Action. American Journal of Sociology, 104(6), 1776-1812.

Kanarattanavong, A. \& Ruenrom, G. (2009). The Model of Corporate Environmentalism: The Effects of Perceived Market Uncertainty upon Marketing, Environmental, and Social Performance. The Business Review, Cambridge, 12(2), 140-147.

Kazley, A. \& Ozcan, Y. (2007). Organizational and environmental determinants of hospital EMR adoption: A national study. Journal of Medical Systems, 31(5), 375-384.

Keil, M. Gordon, D. \& Rai, A. (2007). Escalation: The Role of Problem Recognition and Cognitive Bias. Decision Sciences, 38(3), 391-421.

Khatri, N. \& D'Netto, B. (1997). Perceived Uncertainty and Performance: The Causal Direction. Journal of Applied Management Studies, 6(2), 219-233. 
Kloviene, L. (2013). Improvement of the Performance Measurement System According to Business Environment. Economics and Management, 18 (1), 17-25.

Lawrence, P. \& Lorsch, J. (1967a). Organization and Environment. Managing Differentiation and Integration. Boston: Harvard University.

Lawrence, P. \& Lorsch, J. (1967b). Differentiation and Integration in Complex Organizations. Administrative Science Quarterly, 12(1), 1-47.

López-Gamero, María; Molina-Azorín, J.; \& Claver-Cortés, E. (2011). Environmental Uncertainty and Environmental Management Perception: A multiple. Case Study. Journal of Business Research, 64, 427-435.

Liao, Ch.; Chuang, S. \& To, P. L. (2011). How Knowledge Management Mediates the Relationship Between Environment and Organizational Structure. Journal of Business Research, 64, 728736.

Makridakis, S. (1990). Forecasting, Planning and Strategy for the 21st Century. New York: Free Press.

March, J. \& Simon, H. (1958). Organizations. Las citas corresponden a la versión en castellano: Teoría de la Organización (1987), Ariel Economía.

Miles, R.; Snow, Ch. \& Pfeffer, J. (1974).Organization-Environment: Concepts and Issues. Industrial Relations, 13, 244-264.

Miles, R. y Snow, Ch. (1978). Organizational Strategy, Structure, and Process. McGraw-Hill Publishing Company.

Milliken, F. (1987). Three types of Uncertainty About the Environment: State, Effect and Response Uncertainty. Academy of Management Review, 12(1), 133-143. 
Milliken, F. (1990). Perceiving and Interpreting Environmental Change: An Examin of College Administrator' Interpretation of Changing Demographics. Academy of Management Journal, 33(1), 42-63.

Montanari, J. (1979). Strategic Choice: A Theoretical Analysis. The Journal of Management Studies, 16(2), 202-221.

Mintzberg, H. (1979). The Structuring of Organizations. A Synthesis of the Research. Nueva Jersey: Prentice-Hall.

Mintzberg, H.; Ahlstrand, B. \& Lampel, J. (1998). Strategy Safari. Prentice Hall.

Neisser, U. (1976). Psicología Cognitiva, 1976. Trillas.

Neisser, U. (1981). Procesos Cognitivos y Realidad: Principio e Implicaciones de la Psicología. Editorial Marova.

Nienhüser, W. (2008). Resource Dependence Theory- How Well Does It Explain Behavior of Organizations? Management Revue, 19(1/2), 9-32.

Ogunsiji, A. \& Akanbi, P. (2013).The Role of Perceived Environmental Uncertainty and Strategic Agility on the Performance of Selected Banks in Oyo State of Nigeria. Information and Knowledge Management, 3(7), 23-30.

Parnell, J.; Lester, D.; Long, Z. \& Koseoglu, M. (2012). How Environmental Uncertainty Affects the Link Between business Strategy and Performance in SMEs Evidence from China, Turkey, and the USA. Management Decision, 50(4), 546-568.

Peris, F.; Peris-Ortiz, M. \& Zapata Rotundo, G. J. (2006). Complementariedad de Teorías en la Administración Eficiente del Trabajo: Un Análisis Micro-organizativo. Compendium, 17, 75-94. 
Perrow, Ch. (1970). Organizational Analysis: A Sociological View, en Wadsworth Publishing Company, Inc. Belmont, California.

Pfeffer, J. \& Salancik, G. (1978). The External Control of Organizations: A Resource Dependence Perspective. Harper y Row, Publishers.

Peng, G. \& Beamish, P. (2014). MNC Subsidiary Size and Expatriate Control: Resource-Dependence and Learning Perspectives. Journal of World Business, 49(1), 51-62.

Pissaris, S.; Weinstein, M. \& Stephan, J. (2010). The Influence of Cognitive Simplification Processes on the CEO Succession Decision. Journal of Management Research, 10(2), 71-86.

Rao, M.; Brown, C. \& Perkins, W. (2007). Host Country Resource Availability and Information System Control Mechanisms in Multinational Corporations: An Empirical Test of Resource Dependence Theory. Journal of Management Information Systems, 23(4), 11-28.

Raza, A.; Majeed, A.; Maqsal, M. \& Mansoor, I. (2010). Impact of Resource Based View and Resource Dependence Theory on Strategic Decision Making. International Journal of Business and Management, 5(12), 110-115.

Reitzig, M. \& Sorenson, O. (2013). Biases in the Selection Stage of Bottom-Up Strategy Formulation. Strategic Management Journal, 38, 782-799.

Sawyerr, O.; Ebrahimi, B. \& Thibodeaux, M. (2000). Executive Environmental Scanning, Information Source Utilisation, and Firm Performance: The Case of Nigeria. Journal of Applied Management Studies, 9(1), 95-115.

Schoemaker, P. (1993). Strategic Decision in Organizations: Rational and Behavioural Views. Journal of Management Studies, 30(1), 107-129. 
Schwalb, P.; Leavitt, R.; Barbuto, J.; Millard, M. \& Peetz, R. (2011). Jenny Jan's Dilemma: Applying the Principles of Resource Dependence Theory to Vendor Selection Negotiations. Organization Management Journal, 8, 31-40.

Schwenk, Ch. (1986). Information, Cognitive Biases, and Commitment to a Course of Action. Academy of Management Review, 11(2), 298-310.

Schwenk, Ch. (1988). The Cognitive Perspective on Strategic Decision Making. Journal of Management Studies, 25(1), 41-55.

Scott, C. (2010). Perceptions of Leadership under Conditions of Environmental Uncertainty. The Journal of American Academy of Business, 16(1), 30-35.

Thompson, J. (1967). Organizations in Action. New York: McGrawHill.

Tversky, A. \& Kahneman, D. (1973). Availability: a Heuristic for Judging Frequency and Probability. Cognitive Psychology, 5, 207-232.

Tversky, A. \& Kahneman, D. (1974). Judgement Under Uncertainty: Heuristics and Biases. Science, 185(27), 1124-1131.

Ulrich, D. \& Barney, J. (1984). Perspectives in Organizations: Resource Dependence, Efficiency, and Population. Academy of Management Review, 9(3), 471-481.

Volberda, Henk; Van Der, Weerdt, N.; Verwaal, E.; Stienstra, M. \& Verdu, A. (2012). Contingency Fit, Institutional Fit, and Firm Performance: A Meta fit Approach to OrganizationEnvironment Relationships. Organization Science, 23(4), 1040-1054. 
Walton, E. \& Dawson, S. (2001). Managers' Perceptions of Criteria of Organizational Effectiveness, Journal of Management Studies, 38(2), 173-199.

Weick, K. (1969). The Social Psychology of Organizing. New Jersey: Addison-Wesley Publishing.

Weick, K. (1979). Cognitive Processes in Organization. Research in Organizational Behavior, 1, 47-74.

Weick, K. (1995). Sensemaking in Organizations. Foundations for Organizational Science. A Sage Publication Series, Thousand Oaks.

Weick, K.; Sutcliffe, K. \& Obstfeld, D. (2005). Organizing and the Process of Sensemaking. Organization Science, 16(4), 409-421.

Wei, W. (2006). The Relationship Among Corporate Political Resources, Political Strategies and Political Benefits of Firms in China Based on Resource Dependency Theory. Singapore Management Review, 2(2), 85-14.

Whittington, R. (1988). Environment, Structure and Theories of Strategic Choices. Journal of Management Studies, 25(6), 125-142.

Wong, C., Boon-Itt, S. y Wy-Wong, Ch. (2011). The contingency effects of environmental uncertainty on the relationship between supply chain integration and operational performance. Journal of Operations Management, 29(6), 604-615.

Zabkar, V.; Cater, T.; Bajde, D. y Cater, B. (2013). Environmental Strategy: a Typology of Companies Based on Managerial Perceptions of Customer's Environmental Activeness and Deterrents. E+M Ekonomie a Management, 16(3), 57-74.

Zapata Rotundo, G. y Canet Giner, M. (2009). La Cognición del Individuo: Reflexiones sobre sus Procesos e influencia en la Organización. Revista Espacio Abierto: Cuaderno Venezolano de Sociología, 18(2), 46-62. 
Zapata Rotundo, G. \& Mirabal, A. (2011). El cambio en la organización: Un estudio teórico desde la perspectiva de control externo. Revista Estudios Gerenciales, 27(119), 79-98.

Zapata Rotundo, G. \& Hernández, A. (2014). La Empresa: Diseño, Estructuras y Formas Organizativas. Segunda Edición Ampliada. Caracas: Editorial Horizonte. 


\section{Dr. Gerardo J. Zapata Rotundo}

Licenciado en Contaduría Pública, especialista en Auditoría; con diploma en Estudios Avanzados en Administración de Empresa (DEA); doctor en Dirección de Empresas por la Universidad de Valencia, España. Docente-Investigador Titular del Decanato de Administración y Contaduría (DAC) de la Universidad Centroccidental "Lisandro Alvarado" (UCLA), Venezuela. Posee experiencia docente en las áreas de informática, organización y métodos, sistemas de información y sistemas administrativos. Miembro del Comité Editor de la Revista Científica Compendium (DAC/UCLA). Se desempeña en las líneas de investigación de organización de empresas y teorías de las organizaciones, con diversas publicaciones en revistas científicas, libros y ponencias en congresos. Correo electrónico: zapager@yahoo.com

\section{Dr. Alberto Mirabal}

Licenciado en Ciencias Administrativas; especialista en Desarrollo Organizacional, diploma en Estudios Avanzados en Administración de Empresa (DEA); doctor en Dirección de Empresas por la Universidad de Valencia, España. DocenteInvestigador Titular del Decanato de Administración y Contaduría (DAC) de la Universidad Centroccidental "Lisandro Alvarado" (UCLA), Venezuela. En la actualidad es coordinador del doctorado en Ciencias Administrativas y Gerenciales de la Universidad Centroccidental "Lisandro 
Alvarado". Miembro del Comité Editor de la Revistas Científica Compendium y de Gestión y Gerencia. Sus líneas de investigación son: organización de empresas e internacionalización. Tiene además diversas publicaciones en revistas científicas, libros y ponencias. Correo electrónico: albertomirabal@ucla.edu.ve

\section{Dra. María Teresa Canet Giner}

Licenciada en Ciencias Económicas y Empresariales; doctora en Ciencias Económicas y Empresariales por la Universidad de Valencia, España. En la actualidad es directora del Máster Oficial de Dirección y Gestión de Recursos Humanos, ofertado por la Universidad de Valencia. Como docente e investigadora se enfoca en el área de la Dirección Estratégica y el Diseño Organizativo así como en la aproximación de ambas áreas al estudio de los diversos modelos de excelencia. $\mathrm{Ha}$ centrado también una parte de su investigación en los estudios de género con relación a la estrategia y la negociación, de igual modo estudia los procesos de formación de la estrategia en las Pymes. Correo electrónico: teresa.canet@uv.es

Recibido: 04-03-2015

Aprobado: 07-11-2015 